\title{
A NOTE ON A DIFFERENTIAL CONCOMITANT
}

\author{
P. R. EISEMAN AND A. P. STONE
}

ABSTRACT. If $h$ and $\mathbf{k}$ are vector 1 -forms, the vanishing of the concomitant $[h, k]$ is an integrability condition for certain problems on manifolds. In the case that $h=k$ the vanishing of the Nijenhuis tensor [ $h, h]$ implies $d(\operatorname{tr} \mathbf{h})$ is a conservation law for $\mathbf{h}$, provided that $\operatorname{tr} \mathbf{h}$ is not constant. When the trace of $h$ is constant, a conservation law for $h$ exists if one can find a vector $1 \cdot$ form $k$ with nonconstant trace such that $[h, k]=0$.

1. Introduction. For any vector 1 -form $h$ there is an associated operator $d_{h}$ which is an antiderivation of degree one. In the case that $\mathbf{h}$ is nonsingular and has vanishing Nijenhuis tensor $[\mathrm{h}, \mathrm{h}]$, the operator $d_{h}$ satisfies a Poincaré lemma and thus provides an h-dependent version of de Rham's theorem. Such an operator is an example of a special type of derivation whose basic properties were studied in a definitive paper by $A$. Frölicher and A. Nijenhuis [3]. When $h$ is the identity on 1 -forms, the operator $d_{h}$ reduces to the usual exterior differentiation operator $d_{0}$. This derivation $d_{h}$ and other operators related to it may be used to give an alternative description of the differential concomitant $[h, k]$ of vector 1 -forms $\mathbf{h}$ and $\mathbf{k}$. The vanishing of this concomitant yields the main result of this paper. It is an identity which simply says that the composition ( $d$ otrace) acts as a first order derivation on the composition hk.

The derivational property of $d \circ$ trace is applicable in the study of conservation laws. With it one can establish the existence of conservation laws in a constructive manner which has the added advantage of giving a global result. Since conservation laws have usually been mathematically studied in only a local manner, global results are few. For example, in general relativity the effect of a global conservation law (Einstein's equations) on the manifold topology is of current physical interest (see [2]). Implicit in any global conservation law problem is an interaction with topology; the derivational identity yields a possible method which can be applied in such global problems.

2. Preliminaries. Let $A$ denote the algebra of $C^{\infty}$ functions on a compact, orientable, $n$-dimensional Riemannian manifold $M$ without boundary.

Presented to the Society, January 15, 1974; received by the editors December 9, 1974.

AMS (MOS) subject classifications (1970). Primary 53C15; Secondary 53A55.

Key words and phrases. Vector 1 -form, differential concomitant, differential form, conservation law. 
Let $E$ denote the $A$-module of differential 1 -forms on $M$. A vector 1 -form $h \in$ End $_{A} E$ induces endomorphisms $h^{(q)} \in$ End $_{A}\left(\wedge^{p} E\right)$ for any nonnegative integer $q$, and the $h^{(q)}$ are defined by setting $h^{(q)}=0$ if $q>p$ and

$$
\begin{array}{r}
h^{(q)}\left(\phi^{1} \wedge \cdots \wedge \phi^{p}\right)=\frac{1}{(p-q) ! q !} \sum_{\pi}|\pi|\left\{\mathbf{h} \phi^{\pi(1)} \wedge \cdots \wedge \mathrm{h} \phi^{\pi(q)}\right\} \\
\wedge \phi^{\pi(q+1)} \wedge \cdots \wedge, \phi^{\pi(p)}
\end{array}
$$

if $0 \leq q \leq p$, where $\phi^{i} \in E, \pi$ runs through all permutations of $(1, \ldots, p)$ and $|\pi|$ denotes the sign of the permutation. The transformation $h^{(0)}$ is taken to be the identity mapping on $\wedge^{p} E$.

In the case where $q=p \leq n$, the operator $h^{(p)}$ is locally represented by an $\left(\begin{array}{l}n \\ p\end{array}\right) \times\left(\begin{array}{l}n \\ p\end{array}\right)$ matrix $\left[h^{(p)}\right]$ relative to some local basis of $p$-forms. If [h] denotes an $n \times n$ matrix which locally represents $h$, then it can be shown that

$$
\operatorname{det}\left[h^{(p)}\right]=(\operatorname{det}[h])^{\left(\begin{array}{c}
n-1 \\
p-1
\end{array}\right)}
$$

and hence $\mathbf{h}$ is invertible on 1 -forms if and only if $h^{(p)}$ is invertible on $p$ forms.

An alternating derivation $d_{h}: \wedge E \rightarrow \wedge E$ is obtained from $h$ and an ex॰ terior derivation $d$ by setting $d_{h}=h^{(1)} d-d h^{(1)}$. Thus when $\mathbf{h}$ is the identity, $d_{b}$ reduces to $d$. The Nijenhuis tensor can be extended as a derivation on $\wedge E$. On $p$-forms one thus obtains the formula

$$
[\mathbf{h}, \mathbf{h}]=-b^{(2)} d+d_{h} h^{(1)}+d h^{(2)},
$$

which can be rewritten in the form

$$
[\mathbf{h}, \mathbf{h}]=1 / 2\left\{d_{h^{2}}-\left(d_{h}\right)_{h}\right\} .
$$

In a similar way the concomitant $[\mathrm{h}, \mathrm{k}]$ of vector 1 -forms defined by the equation

$$
\begin{aligned}
& {[\mathbf{h}, \mathbf{k}]=1 / 2\{-} {\left[h^{(1)} k^{(1)}-(h k)^{(1)}\right] d } \\
&\left.+\left[h^{(1)} d k^{(1)}+k^{(1)} d h^{(1)}\right]-\left[d\left[(h k)^{(1)}+k^{(1)} b^{(1)}\right]\right]\right\}
\end{aligned}
$$

can be rewritten in the form

$$
[\mathrm{h}, \mathbf{k}]=1 / 2\left\{d_{h k}-\left(d_{h}\right)_{k}\right\}
$$

3. The main identity. Let $\omega^{1}, \ldots, \omega^{n}$ be a local orthonormal basis of differential 1 -forms and suppose that $\omega^{1} \wedge \ldots \wedge \omega^{n}$ agrees with an orientation of $M$. Suppose also that the vector 1 -form $\mathbf{h}$ is locally specified by setting $\mathbf{h} \omega^{j}=h_{i}^{j} \omega^{i}$, where $h_{i}^{j} \in A$ and $1 \leq i, j \leq n$, and the Einstein summation convention has been invoked. Since the orientation is specified, the Hodge star operator $*^{*} \wedge^{p} E \rightarrow \wedge^{n-p} E$ is determined by setting

$$
*\left(\omega^{i} \wedge \cdots \wedge \omega^{i}{ }^{p}\right)=\epsilon_{i_{1} \cdots_{p}} \omega^{j} \wedge \cdots \wedge \omega^{i} n-p
$$


where $i_{1}<\cdots<i_{p}$ and $j_{1}<\cdots<j_{n-p}$ are complementary sets of positive integers and $\epsilon_{i_{1}} \ldots i_{p}$ is +1 or -1 if the permutation $\left(i_{1}, \ldots, i_{p}, j_{1}, \ldots, j_{n-p}\right)$ of the integers $(1,2, \ldots, n)$ is even or odd respectively. In the sequel the trace of $h$ and the transpose of $h$ will be denoted by $\operatorname{tr} h$ and $h_{t}$ respectively. The following lemma is a key to establishing our identity. The proof of the lemma appears in [1].

Lemma 3.1. For any vector 1 -form $\mathrm{h}$,

$$
h^{(1)} *+* h_{t}^{(1)}=(\operatorname{tr~h}) * .
$$

Since the manifold $M$ is assumed to be compact, an inner product on $\wedge^{p} E$ is defined by setting

$$
(\alpha, \beta)=\int_{M} \alpha \wedge * \beta
$$

for any $p$-forms $\alpha$ and $\beta$. With respect to this inner product the adjoint of $h^{(p)}$ is $h_{t}^{(p)}$ for $p=0,1, \ldots, n$.

The codifferential $\delta$ is defined to be the adjoint of the exterior derivative $d$. The adjoint of $d_{h}=b^{(1)} d-d h^{(1)}$ is then easily seen to be $\delta_{b}=$ $\delta h_{t}^{(1)}-b_{t}^{(1)} \delta$. The operator $\delta_{b}$ is called the h-codifferential. As a consequence of Stokes' theorem one obtains the relation $\delta=(-1)^{n p+n+1} * d *$ on $p$-forms. The corresponding expression for $\delta_{h}$ is obtained in the following lemma.

Lemma 3.2. If $\mathrm{h}$ is a vector 1 -form, then on $p$-forms

$$
\delta_{h}=(-1)^{n p+n+1} *\left\{d_{h}+d(\operatorname{trh}) \wedge\right\} * .
$$

Proof. On $p$-forms one obtains

$$
\delta_{h}=\delta h_{t}^{(1)}-h_{t}^{(1)} \delta=(-1)^{n p+n+1}\left\{* d * h_{t}^{(1)}-h_{t}^{(1)} * d *\right\},
$$

and thus Lemma 3.1 may then be applied to yield

$$
\begin{aligned}
\delta_{h} & =(-1)^{n p+n+1}\left\{* d\left[\operatorname{trh} *-h^{(1)} *\right]-\left[* \operatorname{tr} \mathrm{h}-* h^{(1)}\right] d *\right\} \\
& =(-1)^{n p+n+1}\left\{* d(\operatorname{tr} \mathrm{h}) *-* d h^{(1)} *+* h^{(1)} d *\right\} \\
& =(-1)^{n p+n+1} *\left\{d_{h}+d(\operatorname{tr~h}) \wedge\right\} * .
\end{aligned}
$$

The next lemma establishes a formula for adj[h, $\mathbf{k}]$ for any $\mathbf{h}, \mathbf{k} \in \operatorname{End}_{A} E$; this formula is analogous to the formula for $\delta_{b}$ in the preceding lemma.

Lemma 3.3. For any $\mathrm{h}, \mathrm{k} \in$ End $_{A} E$,

$$
\operatorname{adj}[\mathbf{h}, \mathbf{k}]=(-1)^{n p+1} *\{[\mathbf{h}, \mathbf{k}]+1 / 2\{d(\mathrm{tr} \mathbf{h k})-\mathbf{h} d(\mathrm{tr} \mathbf{k})-\mathbf{k} d(\mathrm{tr} \mathbf{h})\} \wedge \cdot\} *
$$
on p-forms.

\section{Proof. Since}




$$
*[\mathbf{h}, \mathbf{k}]=\frac{1}{2} *\left\{d_{h k}-\left(d_{h}\right)_{k}\right\}=\frac{(-1)^{p(n-p)}}{2}\left\{* d_{h k} *-*\left(d_{h}\right)_{k} *\right\} *
$$

on $p$-forms, it suffices to compute $* d_{h k} *$ and $*\left(d_{h}\right)_{k} *$ on $(n-p) 1$-forms. Thus, as a consequence of Lemma 3.2, one has

$$
* d_{h k} *=(-1)^{n p+1} \delta_{h k}-* d(\mathrm{tr} \mathrm{hk}) *
$$

and

$$
\begin{aligned}
*\left(d_{b}\right)_{k} * & =*\left\{k^{(1)} d_{h}-d_{h} k^{(1)}\right\} * \\
& =\left\{\operatorname{tr~} \mathbf{k}-k_{t}^{(1)}\right\} * d_{b} *-* d_{b} *\left\{\operatorname{tr} \mathbf{k}-k_{t}^{(1)}\right\}
\end{aligned}
$$

by Lemma 3.1. The expression for $*\left(d_{h}\right)_{k} *$ then reduces to

$$
*\left(d_{h}\right)_{k} *=(-1)^{n p+1}\left(\delta_{h}\right)_{k}-*\{\mathbf{k} d(\operatorname{tr} \mathbf{h}) *+\mathbf{h} d(\operatorname{tr} \mathbf{k})\} *
$$

and, hence,

$$
\begin{aligned}
*[\mathbf{h}, \mathbf{k}]= & \left.(-1)^{p+1} \frac{1 / 2}{2} \delta_{h k}-\left(\delta_{h}\right)_{k}\right\} * \\
& +* 1 / 2\{\mathbf{k} d(\operatorname{tr} \mathbf{h})+\mathbf{h} d(\mathrm{tr} \mathbf{k})-d(\mathrm{tr} \mathrm{hk})\} \wedge \cdot .
\end{aligned}
$$

Thus

$$
*[\mathbf{h}, \mathbf{k}]=(-1)^{p+1} \operatorname{adj}[h, k] *+1 / 2 *[\mathbf{k} d(\operatorname{tr} \mathbf{h})+\mathbf{h} d(\mathrm{tr} \mathbf{k})-d(\mathrm{tr} \mathbf{h k})\} \wedge \cdot
$$

and the desired result is then obtained by an application of the Hodge star operator $*$, which is an isomorphism, on the right side of the above expression.

If $[h, k]$ vanishes, then Lemma 3.3 yields an identity which is the main result of this paper. The identity is given in the following proposition.

Proposition 3.4. If $[\mathbf{h}, \mathbf{k}]=0$, then $d(\mathrm{tr} \mathbf{h} \mathbf{k})=\mathbf{h} d(\mathrm{tr} \mathbf{k})+\mathbf{k} d(\mathrm{tr} \mathbf{h})$. Thus $D=d \circ$ trace: $^{\text {End }} A \rightarrow E$ is a first order derivation on compositions of vector $1-$ forms $\mathbf{h}$ and $\mathbf{k}$ whose differential concomitant $[\mathbf{h}, \mathbf{k}]$ vanishes,

One can then study equations of the form $D \mathbf{h}=\alpha$ for some $\alpha \in E$ where a solution $\mathrm{h} \in \mathrm{End}_{A} E$ is desired. This equation is analogous to first order differential equations on the real line. The study of such equations will not be pursued here. However, the fact that $D$ acts analogously to first derivatives on polynomial functions of one real variable will be shown in the following corollaries to Proposition 3.4.

Corollary 3.5. If $[\mathbf{h}, \mathbf{h}]=0$, then for any nonnegative integers $i$ and $j$,

$$
\mathbf{h}^{i} d\left(\operatorname{tr~h}^{j}\right)+\mathbf{h}^{j} d\left(\operatorname{tr~h}^{i}\right)=d\left(\operatorname{tr} \mathbf{h}^{i+j}\right) .
$$

Proof. The vanishing of $[h, h]$ implies the vanishing of the concomitant $\left[\mathrm{h}^{i}, \mathbf{h}^{j}\right.$ ] for any nonnegative integers $i$ and $j$. Thus, as a consequence of Proposition 3.4 with $\mathbf{h}^{i}$ and $\mathbf{h}^{j}$ in place of $\mathbf{h}$ and $\mathbf{k}$, the corollary is established. 
Corollary 3.6. If $[\mathrm{h}, \mathrm{h}]=0$, then for any nonnegative integer $i$, $h^{i} d(\operatorname{trh})=(i+1)^{-1} d\left(\operatorname{tr~h}^{i+1}\right)$.

Proof. If $i=1$, then $\mathrm{h} d(\mathrm{tr} \mathbf{h})=1 / 2 d\left(\mathrm{tr} \mathrm{h}^{2}\right)$ as a consequence of Corollary 3.5. If $\mathbf{h}^{j} d(\mathrm{tr} \mathbf{h})=(j+1)^{-1} d\left(\operatorname{tr} \mathbf{h}^{j+1}\right)$ for some positive integer $j>1$, then

$$
\mathbf{h}^{j+1} d(\mathrm{tr} \mathbf{h})=\frac{1}{j+1} \mathbf{h} d\left(\mathrm{tr} \mathrm{h}^{j+1}\right)=\frac{1}{j+1}\left\{-\mathbf{h}^{j+1} d(\mathrm{tr} \mathbf{h})+d\left(\mathrm{tr}^{\mathbf{h}}{ }^{j+2}\right)\right\}
$$

as another consequence of Corollary 3.5. Hence

$$
\mathbf{h}^{j+1} d(\mathrm{tr} \mathbf{h})=(j+2)^{-1} d\left(\operatorname{tr} \mathbf{h}^{j+2}\right) .
$$

This induction argument establishes the corollary.

Corollary 3.7. If $[\mathrm{h}, \mathrm{h}]=0$, and if $\mathrm{h}$ has constant trace, then the coefficients in the characteristic polynomial of $\mathrm{h}$ are constant,

Corollary 3.7 is an immediate consequence of the fact that if $h$ has constant trace, then all the positive powers of $h$ also have constant trace.

In the case that the coefficients of the characteristic polynomial of a vector 1 -form $h$ are all constant, a result analogous to the formula $\delta *=$ $(-1)^{p+1} * d$ on $p$-forms is obtained. Thus we have

Corollary 3.8. If the coefficients of the characteristic polynomial of $\mathbf{h}$ are constant, then

$$
*[\mathrm{~h}, \mathrm{~h}]=(-1)^{p+1}(\operatorname{adj}[\mathrm{h}, \mathrm{h}]) * .
$$

Proof. Let $\mathrm{h}^{n}=a_{0} I+a_{1} \mathrm{~h}+\cdots+a_{n-1} \mathrm{~h}^{n-1}$ be the characteristic equation of h. Since $a_{n-2}=1 / 2\left\{\operatorname{tr}\left(\mathrm{h}^{2}\right)-a_{n-1}^{2}\right\}$, and $\operatorname{tr} \mathrm{h}=a_{n-1}$, it follows that $\operatorname{tr}\left(\mathbf{h}^{2}\right)$ is constant and, hence, $h d(\operatorname{tr} \mathbf{h})=1 / 2 d\left(\operatorname{tr} \mathbf{h}^{2}\right)=0$. Thus $*[\mathbf{h}, \mathbf{h}]=$ $(-1)^{p+1}$ adj $[h, h] *$ on $p$-forms as a consequence of Lemma 3.3.

This section is concluded with an alternate version of the identity in Corollary 3.6. Since the traces of the various powers of $h$ are expressible in terms of the coefficients $a_{i}$, the following proposition may be obtained.

Proposition 3.9. If $[\mathrm{h}, \mathrm{h}]=0$ and $\mathrm{h}^{n}=a_{0} I+a_{1} \mathrm{~h}+\cdots+a_{n-1} \mathrm{~h}^{n-1}$ is the characteristic equation of $\mathrm{h}$, then

$$
\mathrm{h} d a_{i}=d a_{i-1}+a_{i} d a_{n}, \quad i=0,1, \ldots, n-1\left(d a_{-1} \equiv 0\right) .
$$

Proof. For the case that $h$ is cyclic, a proof appears in [6]. Otherwise the identity in Corollary 3.6 may be used. Thus $h d(\operatorname{tr} \mathbf{h})=1 / 2 d\left(\mathrm{tr} \mathrm{h}^{2}\right)$ and, since $\operatorname{tr} \mathbf{h}=a_{n-1}$ and $\operatorname{tr} \mathbf{h}^{2}=2 a_{n-2}+a_{n-1}^{2}$, one obtains $\mathrm{h} d a_{n-1}=$ $d a_{n-2}+a_{n-1} d a_{n-1}$. Hence, the case $i=n-1$ in the proposition is established. If $\mathbf{h}$ is applied to the above result, the relation obtained is

$$
\begin{aligned}
\mathrm{h} d a_{n-2} & =\mathbf{h}^{2} d a_{n-1}-a_{n-1} \mathrm{~h} d a_{n-1} \\
& =\mathbf{h}^{2} d(\operatorname{tr~h})-a_{n-1} \mathrm{~h} d(\operatorname{tr~h})=(1 / 3) d\left(\mathrm{tr} \mathrm{h}^{3}\right)-\left(a_{n-1} / 2\right) d\left(\mathrm{tr} \mathrm{h}^{2}\right)
\end{aligned}
$$


since $\operatorname{tr} \mathrm{h}^{3}=3 a_{n-3}+3 a_{n-1} a_{n-2}+a_{n-1}^{3}$, the case $i=n-2$ is established. Thus

$$
\mathrm{h} d a_{n-2}=d a_{n-3}+a_{n-2} d a_{n-1}
$$

The remaining identities can be established by repeated use of Corollary 3.6, though the calculation is tedious because of the form of the expressions for $\operatorname{tr} h^{i}$.

Corollary 3.10. If the Nijenhuis tensor $[\mathrm{h}, \mathrm{h}]$ vanishes identically, then the coefficients $a_{i}$ in the characteristic equation for $\mathbf{h}$ are functionally independent if and only $\mathbf{h}$ is cyclic with generator $d(\mathbf{t r} \mathbf{h})$.

Proof. It is sufficient to establish the relation

$$
d(\mathrm{tr} \mathbf{h}) \wedge \mathbf{h} d(\operatorname{tr} \mathbf{h}) \wedge \cdots \wedge \mathbf{h}^{n-1} d(\operatorname{tr} \mathbf{h})=d a_{n-1} \wedge d a_{n-2} \wedge \cdots \wedge d a_{0}
$$

which is a consequence of the proposition.

4. Conservation laws. The conservation laws of physics can be expressed in conservation law form as a differential equation [4], and also as a relation between vector 1 -forms and differential forms [5]. The use of vector 1-forms and differential forms trivially extends the problem to a global one on manifolds. Global conservation laws are of interest in physics as is evident from [2], but to date do not appear to have been studied in a general mathematical context. With the identity stated in Corollary 3.4 the global conservation law problem can be studied.

On a differentiable manifold a conservation law for a vector 1 -form $h$ is defined as any exact differential form $\theta$ such that $h \theta$ is also exact. Thus if $\theta=d f$ for some differentiable function $f$, then $\theta$ is a conservation law for $\mathrm{h}$ if there is a differentiable function $g$ such that $\mathrm{h} d f=d g$. The analysis may be divided into two cases insofar as the existence of conservation laws is concerned. The cases correspond to constant and nonconstant trace for $h$.

If $h$ has trace which is not constant, then Corollary 3.6 says that $d(t r h)$ is a conservation law for any positive power of h. Moreover if $h$ is cyclic with $d(\mathrm{tr} \mathbf{h})$ as a generator, then Corollary 3.1 implies that a basis of conservation laws can be obtained. This discussion is summarized in the following statement.

Proposition 4.1. Let $M$ be a compact orientable n-dimensional Riemannian manifold without boundary and let $\mathrm{h}$ be a vector 1-form whose trace is not constant on $M$. If the concomitant $[\mathbf{h}, \mathbf{h}]$ vanishes on $M$, then $d\left(\mathrm{tr} \mathbf{h}^{i}\right)$ with $i=1,2, \ldots$ is a conservation law for $\mathbf{h}$, and for fixed $i, d\left(\mathbf{t r} \mathbf{h}^{i}\right)$ is a conscruation law for $\mathbf{h}^{j}$, with $j=1,2, \ldots$ Finally if $\mathbf{h}$ is cyclic with generator $d(\mathbf{t r} \mathbf{h})$, then the collection $d\left(\mathrm{tr}^{\mathbf{i}}\right), i=1,2, \ldots, n$, is a basis of conservation laws for $\mathbf{h}$

The analysis for the case in which $\operatorname{tr} h$ is constant is not as simple. 
The above reasoning can be extended provided that a nontrivial solution can be obtained for a certain system of differential equations. The solution of global differential equations is, of course, limited by topological considerations. In particular, if it is possible to find another vector 1 -form $\mathbf{k}$ with nonconstant trace such that the concomitant $[h, k]$ vanishes, then the identity of Proposition 3.4 yields the statement that $d(\mathrm{tr} h)$ is a conservation law for $h$. In local coordinates the vanishing of $[h, k]$ leads to a system of $n\left(\begin{array}{c}n \\ 2\end{array}\right)$ equations (corresponding to $n$ basis elements and $\left(\begin{array}{c}n \\ 2\end{array}\right)$ elements in $E \wedge E$ ) in $n^{2}$ unknowns. This system may be written out explicitly if we assume that $\left(d x^{1}, \ldots, d x^{n}\right)$ is a local basis of 1 -forms, that $\mathbf{h} d x^{i}=h_{\alpha}^{i} d x^{\alpha}$, $\mathbf{k} d x^{i}=k_{a}^{i} d x^{a}$, and that ()$,{ }_{i}=\partial() / \partial x^{i}$. Thus the condition $[\mathbf{h}, \mathbf{k}]=0$ leads to a local system of the form

$$
\begin{aligned}
& 2\left(h_{j}^{\alpha} \delta_{l}^{\beta}-h_{l}^{\beta} \delta_{j}^{\alpha}\right) k_{\beta, a}^{i} \\
& \quad=\left(2 h_{l, j}^{a} \delta_{\beta}^{i}+b_{j, \beta}^{i} \delta_{l}^{\alpha}+b_{\beta_{0} l}^{i} \delta_{j}^{\alpha}-2 h_{j, l}^{\alpha} \delta_{\beta}^{i}-h_{l, \beta}^{i} \delta_{j}^{\alpha}-b_{s, j}^{i} \delta_{l}^{\alpha}\right) k_{\alpha}^{\beta}
\end{aligned}
$$

for $1 \leq j<l \leq n$ and $1 \leq i \leq n$. For $n \geq 3$ the system [h, $\mathbf{k}]=0$ is generally overdetermined but may still have nontrivial solutions. To summarize this section one has

Proposition 4.2. Let $M$ be a compact orientable n-dimensional Riemannian manifold without boundary and let $\mathrm{h}$ be a vector 1-form on $M$. If there is a vector 1 -form $\mathbf{k}$ with nonconstant trace and vanishing concomitant $[\mathbf{h}, \mathbf{k}]$, then $d(\mathrm{tr} \mathbf{k})$ is a conservation law for $\mathbf{h}$, provided $\mathrm{tr} \mathbf{h}$ is constant.

\section{REFERENCES}

1. P. R. Eiseman and A. P. Stone, A generalized Hodge theory, J. Differential Geometry 9 (1974), 169-176. MR 49 \#1534.

2. R. H. Gowdy, Vacuum spacetimes with two-parameter spacelike isometry groups and compact invariant hypersurfaces: topologies and boundary conditions, Ann. Phys. 83 (1974), 208-241.

3. A Frölicher and A. Nijenhuis, Theory of vector-valued differential forms. I. Derivations in the graded ring of differential forms, Nederl. Akad. Wetensch. Proc. Ser. A 59 = Indag. Math. 18 (1956), 338-359. MR 18, 569.

4. P. D. Lax, Hyperbolic systems of conservation laws. II, Comm. Pure Appl. Math. 10 (1957), 537-566. MR 20 \#176.

5. A. P. Stone, Analytic conservation laws, Ann. Inst. Fourier (Grenoble) 16 (1966), fasc. 2, 319-327. MR 35 \#6160.

6. - On the integrability of a vector 1-form, J. London Math. Soc. (2) 6 (1973), 403-411. MR $47 \# 7650$. 06108

UNITED TECHNOLOGIES RESEARCH CENTER, EAST HARTFORD, CONNECTICUT

DEPARTMENT OF MATHEMATICS,UNIVERSITY OF NEW MEXICO, ALBUQUERQUE, NEW MEXICO 87131 